\title{
Das Problem des Nichterscheinens des väterlichen Typus in der Spaltung der partiell sterilen Aquilegia-Species-Bastarde.
}

\author{
Von \\ MARJA SKALIŃSKA.
}

In einer von meinen früheren Abhandlungen (4) habe ich die Erscheinung einer nicht-typischen Mendelschen Spaltung von interspecifischen Aquilegia-Bastarden beschrieben. Reciproke Kreuzungen zwischen den Species Aquilegia vulgaris L. und Aquilegia chrysantha A. Gr. ergeben matrokline Hybride, in deren Nachkommenschaft die erwartete volle Typenreihe in der Spaltung nicht erscheint: wir finden hier nämlich immer den mütterlichen Typus, sowie zahlreiche intermediäre Formen, der väterliche Typus aber ist nicht repräsentiert. Diese Erscheinung einer nicht typischen Spaltung steht im Zusammenhang mit der partiellen Sterilität der Bastarde. Wie ich auf Grund meiner embryologisch-cytologischen Untersuchungen (5) festgestellt habe, degenerieren bei diesen Bastarden bereits gebildete Mikro- und Makrosporen, sowie weibliche Gametophyten und junge Zygoten. In allen diesen Fällen erleiden eine Elimination solche Typen, die zu einer weiteren Entwicklung nicht fähig sind. Man kann vermuten, dass die Ursache solch einer Degeneration in der Haplophase auf eine Dysharmonie zwischen dem Genom, der den väterlichen Typus repräsentiert und dem Cytoplasma, das von der mütterlichen Pflanze stammt, zurückzuführen ist. In der Elimination der Gametophyten können wir auch einen physiologischen Einfluss der mütterlichen Pflanze auf den Haplonten annehmen.

$\mathrm{Da}$ in jeder von beiden reciproken Bastardierungen immer der väterliche Typus ausgeschaltet wird, der mütterliche dagegen neben zahlreichen intermediären Formen erhalten bleibt, so ergibt die zweite 
Hybridengeneration beider reciproken Kreuzungen gesamt betrachtet eine volle Formenreihe in der Spaltung; im Gegenteil, bei einer Analyse der Nachkommenschaft einer jeden von den betreffenden Kreuzungen ein zeln genommen, erweist sich deutlich die Formenreihe in der Spaltung als unvollständig.

Meine bisherigen Untersuchungen sprechen zugunsten der Annahme, dass solch eine nicht typische Spaltung als Konsequenz einer Eliminierung von bestimmten Typen aufzufassen ist, und dass sie stets mit partieller Sterilität verknüpft ist.

In der vorliegenden Arbeit kehre ich nocheinmal zu diesem Problem zurück, auf einem anderen Material gestützt.

Das Problem wird hier auf Grund eines Vergleiches zwischen zwei Bastardierungen erörtert, die sich ausgesprochen durch den Fertilitätsgrad $\operatorname{der} F_{1}$-Pflanzen unterscheiden.

Es werden nämlich die folgenden Kreuzungen besprochen: 1) Aquilegia flabellata nana $\times$ A. chrysantha A. G r. Die erste Generation in dieser Bastardierung ist deutlich matroklin und in hohem Grade steril.

2) Aquilegia flabellata nana $\times A$. californica, sowie die reciproke Kreuzung Aquilegia californica $\times A$. flabellata. Die erste Generation in dieser Bastardierung ist nicht ausgesprochen matroklin und besitzt eine beinahe normale Fertilität.

Der Unterschied in der Fertilität der betreffenden Bastarde muss um desto mehr hervorgehoben werden, dass die beiden Species, die mit derselben $A$. flabellata gekreuzt wurden, $A$. chrysantha und A. californica, eine weitgehende morphologische Ähnlichkeit aufweisen. Deshalb erweisen sich die beiden Bastardierungen als sehr günstige Objekte zur Lösung des obengestellten Problems. Falls ein hoher Sterilitätsgrad als Folge der Elimination des väterlichen Typus aufzufassen ist, so ist bei einer annähernd normalen Fertilität das Erscheinen einer vollen Formenreihe in der Spaltung zu erwarten. Ein Vergleich der zweiten Hybriden-Generation beider Kreuzungen kann also zur Lösung der Frage beitragen, ob die Abwesenheit des väterlichen Typus in der Spaltung tatsächlich durch dessen Elimination verursacht wird, was natürlich zur partiellen Sterilität führen muss.

Es muss hier hervorgehoben werden, dass trotz einer grossen morphologischen Ähnlichkeit zwischen $A$. chrysantha und A. californica, nur in der ersten Bastardierung deutlich matrokline Bastarde entstehen, in der zweiten dagegen können wir solch eine deutliche Prävalenz des mütterlichen Typus in der $F_{1}$-Generation nicht kon- 
statieren. Da aus der ersten Bastardierung Pflanzen mit schwacher Fertilität entstehen, im Gegensatz zu der zweiten, so enthüllt sich daraus noch ein interessantes Problem, nämlich der, ob es ein Zusammenhang zwischen der Matroklinie und der oben erwähnten Elimination des väterlichen Typus existiere und wie wäre er dann zu erklären.

Wenn wir vom „väterlichen“ oder „mütterlichen“ Typus sprechen, so müssen wir ausdrücklich betonen, dass eigentlich solche Termini sich auf Individuen mit einer ganzen Reihe von Merkmalen beziehen, die in der Nachkommenschaft der Bastarde nicht als zusammenhängende Gruppe, sondern als mendelnde Einheiten vererbt werden. Der väterliche resp. der mütterliche Typus ist nur eine von den zahlreichen Kombinationen, die theoretisch die Möglichkeit haben, in der Spaltung der $F_{2}$ Generation rekonstruirt zu werden. Gewisse Störungen, die mit der partiellen Sterilität verbunden sind, können dazu beitragen, nämlich diese Kombination aus der Spaltung auszuschalten, obgleich sie theoretisch zu erwarten ist.

In meinen Untersuchungen jedoch werden die Termini „väterlicher" und "mütterlicher Typus" nicht im Sinne einer präcisen Rekonstruktion der zur Kreuzung bẻnutzten Species gebraucht, sondern in einem etwas engerem Sinne, indem nur einige Merkmale der betreffenden Species in Acht genommen werden, nämlich diejenigen, die in der Spaltung reciproker Bastardierungen Unterschiede aufweisen können. Es sind nämlich: die Form und Länge der Sporne, sowie die Blütenfarben. Die genetische Analyse betrifft hier also nur diese Merkmale.

Zweifellos sind bei Aquilegia-Bastarde für jeden Typus die Form und Länge der Sporne als wichtigste Merkmale zu betrachten. Auf diesen Merkmalen stützt sich übrigens die Klassifikation der beiden Gruppen der Gattung Aquilegia (2). Da aber in gewissen Fällen ein deutlicher Zusammenhang zwischen der Form der Sporne und der Blumenfarbe infolge einer Faktorenkoppelung zu beobachten ist, kann das Erscheinen oder Nichterscheinen von gewissen Farbentypen eine Bedeutung für die genetische Analyse haben. Z. B. im Zusammenhang mit dem Fehlen von geraden langen Spornen (väterlicher Typus) erscheinen äusserst selten Pflanzen mit gelben Blüten in der Spaltung der Bastarde Aquilegia vulgaris $\times A$. chrysantha infolge einer Koppelung des Faktors für diese Blütenfarbe mit demjenigen für die Form der Sporne. Ähnliche Erscheinungen können wir auch in der Spaltung der Kreuzung $A$. flabellata $\times A$. chrysantha beobachten. 


\section{Beschreibung der zur Kreuzung benutzten Species.}

Aquilegia flabellata nana $\mathrm{Sieb}$. und $\mathrm{Zucc}$. Zwergform von 20-25 cm Höhe. Blumen mit gekrümmten kurzen dünnen Spornen mit kleinen Honigdrüsen endend. Die Länge der Honigblätter beträgt $3 \mathrm{~cm}$. Blütenfarbe reinweiss (Fig. 10).

A. chrysantha A. Gr. Pflanze von 60-70 cm Höhe. Blumen mit geraden, dünnen, sehr langen Spornen mit grossen Nektarien endend. Die Länge der Honigblätter beträgt $6.5 \mathrm{~cm}$. Die goldgelbe Blumenfarbe ist durch gelbe Chromoplasten hervorgerufen (Fig. 11).

A. californica $\mathrm{Hartw}$. Pflanze mittelhoch, von ungefähr $45 \mathrm{~cm}$ Höhe. Blumen mit geraden langen Spornen die etwas dicker als diejenigen von $A$. chrysantha sind und mit grossen Nektarien enden. Die Länge der Honigblätter beträgt $6 \mathrm{~cm}$. Die Blüten sind zweifarbig: die Kelchblätter, sowie die Sporne der Honigblätter sind orangerot, während ihr Saum gelb gefärbt ist. Diese Blumenfärbung ist durch zwei Farbstoffe hervorgerufen: durch gelbe Chromoplasten in dem Mesophyll der Blumenblätter und durch das rote Anthocyan, das sich ausschliesslich in den Epidermiszellen im Zellsaft gelöst befindet.

Die beiden letzten Species gehören zu der Gruppe Longicornes (2). Zwischen diesen drei Species wurden folgende Bastardierungen ausgeführt :

1. A. flabellata $\times A$. chrysantha,

2. A. flabellata $\times A$. californica,

3. A californica $\times A$. flabellata.

Die Formen der Sporne bei den Bastarden zwischen A. californica und A. flabellata.

Mittels reciproken Kreuzungen wurden zahlreiche Bastarde zwischen den beiden obengenannten Species erhalten. Es wurde stets eine und dieselbe Californica-Pflanze für alle Kreuzungen benutzt. Sie wurde mit drei Flabellata-Pflanzen von einer völlig einheitlichen Sippe gekreutzt.

Es wurden im Ganzen neun Kreuzungen ausgeführt, nämlich in drei von ihnen wurde $A$. californica als Mutterpflanze genommen, in sechs dagegen wurden mit ihrem Pollen Blumen von A. flabellata bestäubt. Solche Kreuzungen gelingen sehr leicht, und das Erhalten einer zahlreichen $F_{1}$-Generation beider reciproken Bastardierungen bietet keine Schwierigkeiten. 
Es muss hervorgehoben werden, dass die Bastarde, die aus reciproken Kreuzungen stammen, miteinander nicht identisch sind, obgleich man sie nicht streng matroklin nennen kann. lhre Sporne haben in beiden reciproken Kreuzungen die gleiche Länge, sie sind intermediär, und der Unterschied betrifft deren Dicke und Krümmung. Nämlich Bastarde aus der Kreuzung A. flabellata $\times A$. californica haben dünnere Sporne (wie die Mutterpflanze), die aber nur schwach gebogen sind; diejenige der reciproken Bastardierung dagegen besitzen dickere Sporne (wie A. californica), die aber stärker

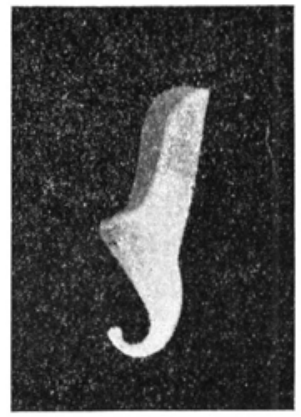

Fig. 10.

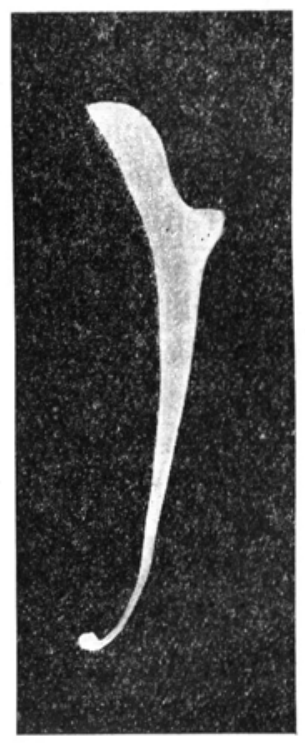

Fig. 11.

gebogen sind, als bei Pflanzen der ersten Kreuzung, im Gegensatz zu den geraden Spornen ihrer Mutterpflanze A. californica. Wir könnten also das Aussehen der Sporne beider reciproken Kreuzungen in diese Weise definieren, dass sie in Bezug auf ihre Länge intermediär, in Bezug auf ihre Dicke - matroklin, in Bezug auf ihre Krümmung aber patroklin sind.

Das Erscheinen ausgesprochen matrokliner Bastarde in reciproken Kreuzungen ist wenigstens scheinbar leichter zu interpretieren, als das Erscheinen solcher Typen, wie die oben erwähnten vorstellen. Eine Prävalenz des mütterlichen Typus bei reciproken Bastarden wird meistens mittels dem Einfluss des Eizell-Plasma erklärt. In dem 
betreffenden Falle aber kann das Problem zweifellos nicht so einfach gelöst werden, da die Krümmung der Sporne eben bei demjenigen Bastarde stärker ausgeprägt ist, dessen Cytoplasma von der geradespornigen $A$. californica stammt. Wir wissen, dass der gekrümmte Sporn über den geraden unvollkommen dominiert, wir könnten deshalb bei beiden reciproken Bastarden im gleichen Grade gekrümmter Sporne erwarten. Würde das Eizellplasma eines Einfluss auf die Ausbildung dieses Merkmals ausüben, so könnten wir in der Kreuzung $A$. flabellata $\times A$. californica stärker gekrümmter Sporne, als in der reciproken Kreuzung erwarten. Da aber, wie gesagt, dieses Merkmal, im Gegenteil, in denjenigen Pflanzen die A. californica als Mutterpflanze haben, deutlicher hervortritt, so ist es ein Beweis, dass sich das Problem nicht so einfach lösen lässt; wir sehen, dass in diesem Falle dieses Merkmal im fremden Plasma zu einer stärkeren Entwicklung gelangt; diese Beobachtung aber gibt uns keineswegs ein Verständnis ihrer Ursachen.

Die Blumen der $F_{1}$-Pflanzen wurden im Frühling 1926 mit Pergamentbeuteln isoliert und geselbstet. Tabelle I gibt uns die Zahlen der gewonnenen Samen für jede Frucht.

\section{Tabelle I.}

\begin{tabular}{|c|c|}
\hline Nr. der Frucht & Anzahl der Samen \\
\hline 1 & 98 \\
2 & 141 \\
3 & 100 \\
4 & 100 \\
5 & 109 \\
6 & 91 \\
7 & 96 \\
8 & 95 \\
9 & 84 \\
10 & 102 \\
\end{tabular}

Trotz einem hohen Fertilitätsgrad der $F_{1}$-Pflanzen konnte ich leider keine zahlreiche $F_{2}$-Generation erhalten. Ein Teil der im Sommer 1926 gereiften Samen des Bastardes A. californica $\times A$. flabellata wurde unmittelbar nach der Ernte ausgesät. Es wurden im Ganzen 350 Samen aus drei Früchten im Juli 1926 ausgesät. Ende August waren die Sämlinge in der Gesamtzahl von 212 Pflanzen in Mistkästen gepflanzt, wo sie überwintern sollten (gewöhnlich keimt ein 
Teil der Samen erst im Frühjahr). Eine beträchtliche Anzahl dieser Pflanzen ging aber wegen dem verspäterten Umpflanzen im Winter zugrunde, während andere zwar am Leben geblieben sind, jedoch im nächsten Frühjahr nicht geblüht haben, was oft als Folge einer verspäterten Saat bei Aquilegia zu beobachten ist. Es war mir leider unmöglich, diese Pflanzen bis zum nächsten Jahr zu behalten, sowie eine wiederholte Aussaat im 1927 oder 1928 zu unternehmen. Deshalb bin ich gezwungen mich mit den Beobachtungen von $1927 \mathrm{zu}$ begnügen. Obgleich aber die Anzahl der in der $F_{2}$-Generation beobachteten Pflanzen gering ist, kann man jedoch konstatieren, dass im Gegensatz zu den früher beschriebenen Bastarden zwischen $A$. vulgaris und $A$. chrysantha, in $\operatorname{der} F_{2}$-Generation dieser Kreuzung der väterliche Typus der Sporne in der Spaltung erscheint. Wie aus der Tabelle II zu schliessen ist, treten Pflanzen mit intermediären Längen der Sporne am zahlreichsten auf (53 Individuen, was $65 \%$ aller Individuen bildet). Beide extremen Typen - der väterliche und der müttërliche, derjenige mit langen und mit kurzen Spornen, sind auch in der Spaltung zu finden, wobei diese letzteren zahlreicher, als die ersten auftreten. In Bezug auf die Krümmung der Sporne findet ebenfalls eine Spaltung statt, und zwar in gerade, gebogene und gekrümmte Sporne. Wie aus der Tabelle II ersichtlich ist, werden verschiedene Kombinationen der Länge mit der Form der Sporne gebildet; es entstehen lange gekrümmte Sporne, sowie auch kurze gerade Sporne, was in der Kreuzung A. flabellata $\times A$. chrysantha nicht stattfindet.

\section{Tabelle II.}

\begin{tabular}{|c|c|c|c|c|c|}
\hline \multirow{2}{*}{$\begin{array}{c}\text { Form der } \\
\text { Sporne der } \\
F_{2} \text {-Pflanzen } \\
\text { A. californica } \\
\times \text { A. flabellata }\end{array}$} & \multicolumn{5}{|c|}{ Länge der Sporne der $F_{2}$-Pflanzen } \\
\hline & $\begin{array}{c}\text { I. } \\
\text { Wie A.cali- } \\
\text { fornica }\end{array}$ & \begin{tabular}{|c|} 
II. \\
Etwas kürzer \\
als $A$. cali- \\
fornica \\
\end{tabular} & $\begin{array}{c}\text { III. } \\
\text { Intermediär }\end{array}$ & \begin{tabular}{|c|} 
IV. \\
Etwas länger \\
als $A$. flabel- \\
lata
\end{tabular} & $\begin{array}{c}\text { V. } \\
\text { Wie } \begin{array}{c}\text { A. flabel- } \\
\text { lata }\end{array}\end{array}$ \\
\hline $\begin{array}{c}\text { A } \\
\text { Gerade } \\
\text { Sporne }\end{array}$ & $\begin{array}{c}\text { Typus A I } \\
\left(\begin{array}{c}\text { A. californica }) \\
4 \text { Pflanzen }\end{array}\right.\end{array}$ & $\begin{array}{l}\text { Typus A II } \\
15 \text { Pflanzen }\end{array}$ & $\begin{array}{l}\text { Typus A III } \\
5 \text { Pflanzen }\end{array}$ & $\begin{array}{c}\text { Typus A IV } \\
1 \text { Pflanze }\end{array}$ & $\begin{array}{l}\text { Typus A V } \\
2 \text { Pflanzen }\end{array}$ \\
\hline $\begin{array}{c}\mathrm{B} \\
\text { Gebogene } \\
\text { (intermediäre) } \\
\text { Sporne }\end{array}$ & $\begin{array}{l}\text { Typus B I } \\
8 \text { Pflanzen }\end{array}$ & $\begin{array}{l}\text { Typus B II } \\
9 \text { Pflanzen }\end{array}$ & $\begin{array}{l}\text { Typus B. III } \\
13 \text { Pflanzen }\end{array}$ & $\begin{array}{l}\text { Typus B IV } \\
4 \text { Pflanzen }\end{array}$ & $\begin{array}{l}\text { Typus B V } \\
3 \text { Pflaṇzen }\end{array}$ \\
\hline $\begin{array}{c}\text { C } \\
\text { Gekrümmte } \\
\text { Sporne }\end{array}$ & $\begin{array}{c}\text { Typus C I } \\
1 \text { Pflanze }\end{array}$ & $\begin{array}{l}\text { Typus C II } \\
2 \text { Pflanzen }\end{array}$ & $\begin{array}{l}\text { Typus C III } \\
4 \text { Pflanzen }\end{array}$ & $\begin{array}{l}\text { Typus C IV } \\
0 \text { Pflanzen }\end{array}$ & \begin{tabular}{|l} 
Typus C V \\
$(A$ flabellata $)$ \\
10 Pflanzen
\end{tabular} \\
\hline
\end{tabular}


Ich muss betonen, dass die hier beobachteten Zahlenverhältnisse vielleicht nicht völlig zuverlässig sind, da eigentlich nur ein Teil der Aussaat mir zur Verfügung stand. Jedoch handelt es sich hier hauptsächlich nicht um die Zahlen, in denen die verschiedenen Typen vertreten sind, sondern um die Anwesenheit gewisser Typen, was für das erörterte Problem von prinzipiellen Bedeutung ist. Wir konstatieren also, dass in der Nachkommenschaft der fertilen Bastarde A. californica $\times A$. flabellata die beiden Elterntypen in der Spaltung erscheinen; im Zusammenhang mit der Fertilität ist die Typenreihe der Spaltung vollständig.

Formen der Sporne der Bastarde A. flabellata $X$ A. chrysantha.

Die nur aus einer Kreuzung $A$.flabellata $\times$ A.chrysantha gewonnene Bastarde in der Gesamtzahl von 30 Pflanzen erwiesen sich als matroklin in Bezug auf die Form und Länge ihrer Sporne. Die Sporne übertrafen nämlich nur schwach in ihrer Länge diejenigen von $A$. $f a$ bellata, sie waren schwächer gekrümmt und besassen kleine Nektarien, was auch ein Merkmal von A. flabellata ist. Diese Prävalenz des mütterlichen Typus ist an den Dimensionen der Sporne beider Elterpflanzen und der Hybride deutlich sichtbar:

Die Länge der Honigblätter von A. flabellata beträgt $3.0 \mathrm{~cm}$

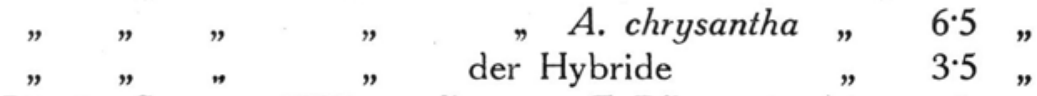

Die im Sommer 1925 geselbsteten $F_{1}$-Pflanzen erwiesen eine sehr schwache Fertilität nach der Selbstbestäubung (Tabelle III). Die Sa men wurden im Frühling 1926 gesät. Nicht alle Samen waren kei-

Tabelle III.

\begin{tabular}{|c|r|}
\hline Nr. der Frucht & Anzahl der Samen \\
\hline 1 & 11 \\
2 & 12 \\
3 & 33 \\
4 & 3 \\
5 & 10 \\
6 & 2 \\
7 & 10 \\
8 & 7 \\
9 & 29 \\
10 & 23 \\
& \\
\hline
\end{tabular}


mungsfähig. Viele von den gekeimten Pflanzen zeigten eine abgeschwächte Entwicklung und waren noch kleiner und bedeutend zarter, als die Zwergform A. flabellata. Neben einer Anzahl normaler Individuen, fand man viele Pflanzen, die Degenerationstypen vorstellten. Manche von ihnen ergaben nur eine einzige Blüte, andere blühten überhaupt nicht, obgleich sie frühzeitig gesät und umgepflanzt wurden, und sie gingen in der zweiten Vegetationsperiode zugrunde. Deshalb sind die mir zur Verfügung stehende Zahlen der $F_{2}$-Generation gering.

\section{Tabelle IV.}

\begin{tabular}{|c|c|c|c|c|c|}
\hline \multirow{2}{*}{$\begin{array}{c}\text { Form der } \\
\text { Sporne der } \\
F_{2} \text {-Pflanzen } \\
\text { A. flabellata } \times \\
\text { A. chrysantha }\end{array}$} & \multicolumn{5}{|c|}{ Länge der Sporneder $F_{2}$-Pflanzen } \\
\hline & $\begin{array}{l}\text { I. } \\
\text { Wie } A . \text { chry- } \\
\text { santha }\end{array}$ & \begin{tabular}{|c|} 
II. \\
Etwas kürzer \\
als $A$. chry- \\
santha
\end{tabular} & $\begin{array}{c}\text { II. } \\
\text { Intermediär }\end{array}$ & $\begin{array}{c}\text { IV. } \\
\text { Etwas länger } \\
\text { als A. flabel- } \\
\text { lata }\end{array}$ & $\begin{array}{c}\text { V. } \\
\text { Wie A. flabel- } \\
\text { lata }\end{array}$ \\
\hline $\begin{array}{c}\text { A } \\
\text { Gerade } \\
\text { Sporne }\end{array}$ & $\begin{array}{l}\text { Typus A I } \\
(A . \text { chry- } \\
\text { santha }) \\
0 \text { Pflanzen }\end{array}$ & $\begin{array}{l}\text { Typus A II } \\
3 \text { Pflanzen }\end{array}$ & $\begin{array}{l}\text { Typus A III } \\
5 \text { Pflanzen }\end{array}$ & $\begin{array}{c}\text { Typus A IV } \\
1 \text { Pflanze }\end{array}$ & - \\
\hline $\begin{array}{c}\text { B } \\
\text { Gebogene } \\
\text { (intermediäre) } \\
\text { Sporne } \\
\end{array}$ & - & $\begin{array}{l}\text { Typus B II } \\
3 \text { Pflanzen }\end{array}$ & $\begin{array}{l}\text { Typus B III } \\
7 \text { Pflanzen }\end{array}$ & $\begin{array}{l}\text { Typus B IV } \\
6 \text { Pflanzen }\end{array}$ & - \\
\hline $\begin{array}{c}\text { C } \\
\text { Gekrümmte } \\
\text { Sporne }\end{array}$ & - & - & $\begin{array}{c}\text { Typus C III } \\
1 \text { Pflanze }\end{array}$ & $\begin{array}{l}\text { Typus C IV } \\
6 \text { Pflanzen }\end{array}$ & $\begin{array}{l}\text { Typus C V } \\
\text { (A. flabellata) } \\
6 \text { Pflanzen }\end{array}$ \\
\hline
\end{tabular}

Aus der Tabelle IV ist es ersichtlich, dass Pflanzen vom mütterlichen Typus der Sporne in der Spaltung repräsentiert sind, dass dagegen der väterliche Typus fehlt; es erscheinen jedoch Pflanzen, die ihm in der Form der Sporne nahe stehen. Am ähnlichsten der A. chrysantha sind Pflanzen vom Typus $A I I$ (Tabelle IV) sie besitzen aber etwas kürzere Sporne, sie repräsentieren also nicht die genaue Rekonstruktion des väterlichen Typus. Wie schon oben erwähnt wurde, trug eine bedeutende Anzahl der $F_{2}$-Pflanzen deutliche anzeichen der Degeneration und kam überhaupt nicht zur Blüte. Im Gegenzatz zu diesen Formen, erscheinen intermediäre Formen sowie auch Pflanzen vom mütterlichen Typus im allgemeinen kräftig und normal entwickelt. Man muss bemerken dass in dieser Kreuzung zwischen der Länge der Sporne und deren Krümmung eine deutliche negative Korrelation zu beobachten ist. Je länger die Sporne, desto weniger gebogen, je kürzer sie sind, desto deutlicher die Krümmung. 
Das Erscheinen des mütterlichen Typus und das gleichzeitige Fehlen des väterlichen Typus in der Nachkommenschaft von Hybriden zwischen entfernte Species ist eine Bestätigung unserer früheren Beobachtungen über reciproke Bastarde zwischen $A$. vulgaris und A. chrysantha. Wahrscheinlich bleibt diese Erscheinung auch hier in Zusammenhang mit der partiellen Sterilität.

\section{Blütenfarben der Hybride.}

Die Blütenfarben der beiden hier besprochenen Bastardierungen weisen ziemlich verwickelte Verhältnisse auf. Sie sind schwer zu analysieren ohne die Kenntnis von einer Reihe Beobachtungen, die andere Aquilegia-Kreuzungen betreffen und die mir die Gelegenheit gegeben haben, die Phänomene der Synthese und der Spaltung der Blütenfarben verständlich zu machen. Deshalb müssen die Resultate jener Beobachtungen hier im Kurzen wiedergegeben werden.

Bastardierungen zwischen verschiedenen Arten der Gattung Aquilegia, welche kein Anthocyan in ihren Blüten enthalten, führen äusserst oft zu einer Synthese von Anthocyanpigmenten in $F_{1}$. Auch infolge einer Bastardierung zwischen Pflanzen mit weissen und farbigen Blüten konstatieren wir oft eine Veränderung der Blütenfärbung bei den Bastarden.

Meine Beobachtungen, die einen umfangreichen Material von Artkreuzungen umfassen, erlaubten mir festzustellen, daß zahlreiche Species, deren Blumen kein Anthocyan enthalten, jedoch in ihrer genetischen Konstitution latent bleibende Faktoren besitzen, die in bestimmten, infolge der Kreuzung entstehenden Kombinationen, zur Synthese des Anthocyans führen können. Ausser solcher Faktoren finden wir auch bei gewissen weiss- resp. gelbblühenden Species latente Faktoren, die bei Kreuzung mit rotblühenden Species, die rote Anthocyafärbung bei den Bastarden ins blaue resp. violette verändern. Ähnliche Faktoren sind schon längst bei Lathyrus odoratus dank den Untersuchungen von Bateson und Punnett (1) bekannt. Ihre Wirkung beruht wahrscheinlich auf einer Neutralisierung der saueren Reaktion des Zellsaftes.

Indem wir also nicht nur die äusserlich sichtbaren Merkmale in Acht nehmen, sondern in die genetische Konstitution der Pflanzen mit der Berücksichtigung von latenten Faktoren, tiefer eindringen, so können wir bezüglich der Faktoren der Blumenfärbung die Gattung Aquilegia in zwei grosse Gruppen teilen:

A) Pflanzen mit violetten, blauen und weissen Blüten mit neutraler Reaktion des Zellsaftes. Die violette resp. blaue Blumenfarbe 
„dominiert" über rote und rosa Färbung, was schon von Krist offerson (3) hervorgehoben wurde. Diese "Dominanz" beruht auf der Anwesenheit eines Faktors, dessen Wirkung wahrscheinlich die Reaktion des Zellsaftes neutralisiert. Pflanzen mit we is s e n Blumen, die ebenfals zu dieser Gruppe gehören können, ergeben, falls sie mit rot oder rosablühenden Pflanzen gekreuzt werden, Pflanzen mit violeten oder blauen Blumen; falls die Kreuzung solcher Pflanzen mit irgendwelchen andern Species ohne Anthocyan zur Synthese des Anthocyans führt, so entsteht auf diesem Wege blaues oder violettes, niemals aber rotes Anthocyan. Zu diese Gruppe zähle ich auf Grund der durchgeführten Kreuzungen folgende Species:

A. vulgaris mit blauen und violetten Blüten.

A. Olympica (A. Wittmanniana) mit blauen Blüten, sowie deren Varietät mit weissen Blüten, die Spuren von blauen Anthocyan in den Spornen enthalten.

A. flabellata.

$B)$ Pflanzen mit roten, orangeroten, gelben und weissen Blumen mit saurer Reaktion des Zellsaftes. Die verschiedenen Nuancen der roten Farbe sind gegen violett und blau recesiv. Zu diesser Gruppe gehören folgende Species die in meinen Experimenten zur Kreuzung benutzt wurden: $A$. californica, $A$. truncata $\mathrm{F}$ is ch. und $\mathrm{Mey}, A$. Skinnerii Hook, A. chrysantha A. Gr., A. chrysantha, var. alba, $A$. olympica weissblühende Varietät mit Spuren von hellrosa Anthocyan an der Basis der Blumenblätter, A. azurea hort, fl. roseo, A. vulgaris, Varietät mit hellrosa Blumen. Die drei letzteren sind Varietäten, die höchstwahrscheinlich von Species der ersten Gruppe stammen und von ihnen vermutlich auf dem Wege der Mutation oder Kreuzung entstanden sind.

Auf Grund meiner Experimente stellte ich fest, dass Kreuzungen zwischen zwei Anthocyan enthaltenđen Vertretern dieser beiden Gruppen unbedingt zu einer $F_{1}$-Generation mit violetten oder blauen Blumen führen. Auch wenn wir zwei weißblühende Pflanzen, von denen jede zu einer anderen der beiden erwähnten Gruppen gehört, miteinander kreuzen, so beobachten wir die Synthese des blauen Anthocyans. Solch ein Resultat erhalten wir z. B. infolge der Bastardierung zwischen A. flabellata und der weissblühenden Varietät (mit Spuren von rosagefärbtem Anthocyan) von A. olympica. Dasselbe Resultat bekommen wir, indem wir $A$. chrysantha (resp. A. chrysantha, var. alba), mit $A$. flabellata kreuzen. Die Kreuzung von we is s blühenden Pflanzen der Gruppe $A$ mit farbigen (rot und rosagefärbten) Blumen von den 
Species der Gruppe $B$ führt immer zur Bildung von violett oder blau blühenden Hybriden. $A$. californica und $A$. truncata mit $A$. flabellata gekreuzt, geben violettblühende Bastarde. Aus der Kreuzung von $A$. flabellata mit $A$. vulgaris $f l$. roseo und $A$. azurea $f l$. roseo entstehen Pflanzen mit blauen Blüten.

Auch eine Kreuzung weissblühender Species im Gebiete der Gruppe $A$ kann zu einer Synthese des Anthocyans führen, der entstende Farbstoff hat dann immer eine blaue oder violette Färbung. So z. B. die weisse Varietät von $A$. olympica, die sich von der Stammart durch das Fehlen wenigstens eines Faktors, der zur Synthese des Anthocyans beiträgt unterscheidet, ergibt mit A. flabellata gekreuzt, eine Rekonstruktion des blauen Anthocyans. Daraus kann man schliessen, dass die $\mathrm{Zahl}$ der Faktoren die zur Synthese des Anthocyans beitragen, hier wenigstens zwei ist, ohne den Faktor für Farbenveränderung zu rechnen.

Auch im Gebiete der zweiten Gruppe können Kreuzungen eine Synthese des Anthocyans in $F_{1}$ verursachen; jedoch ist der entstehende Pigment immer rosa (d. h. heterozygot rot) niemals aber violett oder blau. Z. B. die Kreuzung von A. chrysantha mit der weissblühenden Varietät mit Anthocyansspuren von $A$. olympica ergibt Bastarde mit deutlich rosa gefärbten Blumen mit gelbem Saum der Honigblätter.

Endlich muss man bemerken, dass im Gebiet der zweiten Gruppe ausgeführte Kreuzungen zwischen weissblühenden Species und solchen, deren Blumen rotes Anthocyan enthalten, niemals eine Modifikation der roten Farbe in eine violette oder blaue verursachen, sondern bei den Bastarden nur eine Abschwächung der Färbungsintensität hervorruffen, da selbstverständlich die betreffenden Faktoren bei den Bastarden heterozygotisch auftreten. So z. B. nach der Kreuzung von $A$. azurea var alba mit $A$. californica erhalten wir rosablühende $F_{1}$ - Pflanzen mit hellgelbem Saum der Honigblätter, was annährend $\operatorname{der} A$. azurea $f l$. roseo entspricht. In $\operatorname{der} F_{2}$ Generation beobachtet man bei völlig typischer, ziemlich einfach verlaufenden Spaltung, ein Rekonstruktion der beiden Elternformen.

Die zwei Kreuzungen, die in dieser Arbeit eingehend besprochen werden, sind eben solche Bastardierungen zwischen den Vertretern der beiden oben erwähnten Gruppen. Nämlich $A$. flabellata gehört zu der ersten Gruppe, und $A$ californica sowie $A$. chrysantha zu den zweiten. Meine früheren Untersuchungen, die nur teilweise publi- 
ciert wurden, können gewissermassen zur Kenntnis der genetischen Konstitution von $A$. californica beitragen. Nämlich in der Spaltung der Bastarde $A$. chrysantha $\times$ A. vulgaris (4), erschienen Pflanzen mit orangeroten Blumen und geraden Spornen; die Blumen solcher Pflanzen ähneln völlig denjenigen von $A$. californica; sie enstehen infolge der Anwesenheit der Faktoren für gelbe Blumenfärbung ( $Y$ ) in Verbindung mit denjenigen für die Bildung des Anthocyans, wobei der Faktor $F$, welcher die Reaktion des Zellsaftes verändert, abwesend ist. Auf Grund des Erscheinens solcher Pflanzen in der $F_{2}$ der erwähnten Kreuzung können wir schliessen, dass in violetten Blumen von $A$. vulgaris und in orangenroten Blumen von A. californica eingentlich dasselbe Anthocyan vorhanden ist, dessen Farbe von der Reaktion des Zellsaftet abhänging ist. A. chrysantha deren Blumen kein Anthocyan führen, unterscheidet sich von $A$. californica durch die Abwesenheit von gewissen Faktoren, die zur Synthese des Anthocyans notwendig sind; beiden Species jedoch sind gewisse Charaktere gemeinsam, nämlich der Besitz gelber Chromoplasten in den Geweben der Blumen, sowie die saure Reaktion des Zellsaftes. Auch eine grosse Ähnlichkeit der Form der Sporne muss hervorgehoben werden. Der obige Vergleich lässt uns die Möglichkeit einer tatsächlichen Verwandschaft zwischen diesen zwei in Kalifornien einheimischen Species vermuten.

Ausser dem eben erwähnten Erscheinen von Pflanzen, die eine Rekonstruktion der A. californica vorstellen, seien noch andere Beobachtungen angeführt, die die genetische Analyse der Blumenfarbe von $A$. californica ermöglichen. Nämlich bei der Bastardierung dieser Species mit $A$. azurea hort. var. alba, bekommen wir in $F_{1}$ rosablühende Pflanzen mit hellgelbem Saum der Honigblätter. Die Intensität beider Pigmente der Blumen von A. californica wird hier bedeutend abgeschwächt; indessen muss es hervorgehoben werden, dass diese Abschwächung das gelbe Pigment in noch stärkerem Grade betrifft, als das Anthocyan. Deshalb ist die Blumenfarbe nicht hellorangenrot, sondern deutlich hellkarminrot. Die auf dem Wege des Selbstbestäubung völlig fertiler $F_{1}$-Bastarde dieser Kreuzung gewonnene Nachkommenschaft gibt eine einfache regelmässig verlaufende Spaltung von zwei Faktorenpaaren $R$ und $Y$. In der Spaltung erscheinen die beiden älterlichen Typen ( $R R y y$ und rryy), sowie Formen, die verschiedene Kombinationen dieser Faktoren vorstellen, nämlich $R R y y$ und $R R Y_{y}$-Pflanzen mit karminroten Blumen mit weiss und hellgelb gesäumten Honigblätter; Rryy und $\operatorname{Rr} Y y$-Pflanzen mit karminrosa Blumen mit weiß und hellgelb gesäumten Honigblätter; $r r Y Y$ und 
$r r Y y$ - Pflanzen mit gelben und hellgelben Blumen. Ich erwähne hier diese Beobachtungen, die bisher unveröffentlich geblieben sind, da sie zweifellos für die Kreuzungsanalyse von $A$. californica einen Wert haben können.

Die erste Bastardengeneration zwischen $A$. flabellata und $A$. californica besitzt violette Blumen (C. C. ${ }^{1}$ ) 526-528) mit hellgelbem Saum der Honigblätter. Unter dem Einfluss des oben erwähnten Faktors, dessen Wirkung als Modifikation der Reaktion des Zellsaftes aufzufassen ist und der durch $A$ flabellata in die Kreuzung eingeführt wird, erscheint eine Farbenveränderung des orangenroten Anthocyans ins violette.

Die Spaltung der zweiten Generation verläuft in Allgemeinen typisch, obgleich sie ziemlich kompliciert ist (Tabelle VI). In $\operatorname{der} F_{2^{-}}$ Generation erscheinen folgende Farbentypen:

1. Pflanzen mit violetten und rotvioletten Blumen (C. C. 526, 528, 536, 546, 557, 561 und 582) in der Zahl von 53 Individuen.

2. Pflanzen mit orangenroten Blumen mit gelbem Saum der Honigblätter (wie A. californica) in der Zahl von 3 Individuen.

3. Pflanzen mit hellorangenroten Blumen mit gelbem Saum der Honigblätter in der Zahl von 3 Individuen.

4. Pflanzen mit karminroten Blumen mit hellgelbem Saum der Honigblätter in der Zahl von 4 Individuen.

5. Pflanzen mit rosa Blumen mit hellgelbem Saum der Honigblätter in der Zahl von 4 Individuen.

6. Pflanzen mit rosa Blumen in der Zahl von 3 Individuen.

7. Pflanzen mit gelben und hellgelben Blumen in der Zahl von 3 Individuen.

8. Pflanzen mit weissen Blumen in der Zahl von 8 Individuen.

In der Spaltung der $F_{2}$-Generation entspricht ein beträchtlicher Teil der Pflanzen, ihrer Blumenfarbe nach, der $F_{1}$-Generation. Ich vermute, dass diese Zahlenverhältnisse eigentlich nicht völlig zuverlässig sind, da sie wahrscheinlich die Folge einer Selektion zwischen den $F_{2}$-Pflanzen sind. Jedoch trotz der geringen Anzahl der Pflanzen ist es gewissermaassen möglich, sich in den mendelnden Einheiten zu orientieren, wenn wir die oben erwähnten Angaben zu Hilfe nehmen. Wie gesagt, besitzt $A$. californica in ihren Blumenblätter zwei verschiedenen Farbenstoffe, nämlich den gelben mit Chromoplasten verbundenen Farbstoff, der vom Faktor $Y$ bedingt ist, sowie das

1) Klinksieck \& Valette: Code des Couleurs. Paris 1908. 
Anthocyan, dessen Synthese wenigstens von zwei Faktoren verursacht ist. Diese Auffasung ist hoffentiich auf Grund des Obengesagten genügend bewiesen worden. Der Faktor $R$ der früher erwähnt wurde, ist also nicht der einzige Faktor der das Erscheinen des Anthocyans bedingt; infolge einer Zusammenwirkung dieses Faktors mit einem anderen $(C)$ findet die Synthese des Anthocyans statt.

Was dagegen die genetische Konstitution der A. flabellata betrifft, so wissen wir auf Grund des Obengesagten, dass sie einen Faktor enthält, der die Farbenänderung des Anthocyans verursacht. Ausserdem enthält auch diese Pflanze einen von den beiden Faktoren, die die Synthese des Anthocyans bedingen. Die Resultate der Kreuzung dieser Pflanze mit $A$. chrysantha sprechen zugunsten dieser Annahme. Nämlich da die $F_{1}$-Pflanzen dieser Kreuzung in ihren Blumen Anthocyan enthalten, so können wir daraus schliessen, dass A. flabellata denjenigen der beiden Faktoren enthält, der in A. chrysantha fehlt, nämlich der Faktor $R$, während $C$, aller Wahrscheinlichkeit nach, in $A$. chrysantha enthalten ist.

Die Spaltung in der $F_{2}$-Generation von $A$. californica $\times A$. flabellata würde also drei Faktorenpaare betreffen, da der Faktor $R$ hier homozygot erscheint, weil er in beider Elternpflanzen enthalten ist. Die obenerwähnten Farbentypen, die in der Spaltung erscheinen, würden nach dieser Voraussetzung folgende Kombinationen der genetischen Faktoren vorstellen:

1. Pflanzen mit violetten und rotvioletten Blumen bilden eine nicht einheitliche Gruppe, die Individuen mit dunkleren und helleren Blumen umfasst. Ihre Farbennuancen erscheinen mehr violett oder mehr rotviolett, ohne dass eine deutliche Grenze zwischen ihnen durchgeführt werden kann. Die Färbung dieser Blumen hängt von den Faktoren $C, R$ und $F$ ab; eine dunklere Färbung erscheint bei Homozygoten, eine hellere dagegen bei Heterozygoten. Deutlichere violette Nuancen sind wahrscheinlich mit homozygotem Zustand des Faktors $F$ verbunden, infolgedessen sich der Process der veränderung des roten Anthcyans ins violette in vollständiger Weise abspielen würde; die rotviolette Färbung dagegen würde mit heterozygotem $F$ verbunden sein. Ausser dem Anthocyan, dessen Anwesenheit und Farbe vom Spiel der drei obengenannten Faktoren abhängig ist, erscheint in den Blumen von gewissen Pflanzen dieser Gruppe das gelbe Pigment, das durch das Anthocyan verdeckt ist und dessen Anwesenheit nur an der gelben Färbung des Saumes der Honigblätter zu erkennen ist. 
2. Pflanzen mit orangenroten Blumen mit gelbem Saum der Honigblätter geben die Rekonstruktion des mütterlichen Farbentypus A. californica, sie enthalten die Faktoren $C C R R f f Y Y$.

3. Pflanzen mit hellorangenroten Blumen mit gelbem Saum der Honigblätter stehen nahe der vorigen Gruppe, mit dem Unterschied dass der Faktor $C$ heterozygot ist. Infolgedessen ist die Intensität des Anthocyans, das auf $\mathrm{e}$ inem deutlichen gelben Hintergrund hervortritt, sehr abgeschwächt.

4. Pflanzen mit karminroten Blumen mit hellgelbem Saum der Honigblätter, besitzen im homozygotem Zustand die beiden Faktoren $C R$ für die Anthocyanbildung in Abwesenheit von $F$; dagegen $Y$ ist heterozygot, wodurch seine Wirkung deutlich abgeschwächt ist.

5. Pflanzen mit rosagefärbten Blumen mit hellgelbem Saum der Honigblätter stehen nahe der vorigen Gruppe, nur ist der Faktor $C$ heterozygot, was eine weniger intensive Färbung des Anthocyans bedingt.

6. Pflanzen mit hellrosa Blumen ohne gelben Chromoplasten. Ihre Färbung hängt von der Zusammenwirkung von zwei Faktoren $R$ und $C$ ab, von denen der erste homozygot, der zweite heterozygot ist; die Faktoren $F$ und $Y$ sind abwesend.

In der Spaltung könnte man eigentlich noch Homozygoten mit karminroten Blumen ohne gelben Chromoplasten (von der Formel $R K C C f f y y)$ erwarten, jedoch sind solche Pflanzen, deren Erschei, nen nur einmal auf 64 Kombinationen zu erwarten ist, in der Spaltung nicht erschienen.

7. Pflanzen mit gelben und hellgelben Blumen. Ihre Färbung ist durch den Faktor $Y$ der in den ersten homozygot, in den zweiten heterozygot auftritt, verursacht, während $C$ abwesend ist und $F$ (falls vorhanden) latent bleibt.

8. Pflanzen mit weissen Blumen bilden eine Gruppe die hinsichtlich ihrer Blumenfarbe einheitlich, aber ihrer Genotypus uneinheitlich ist. Alle diese Pflanzen besitzen homozygot den Faktor $R$ in Abwesenheit von $C$, und eine Anzahl Pflanzen dieser Gruppe besitzt auch latent den Faktor $F$. Zwischen den weissblühenden Pflanzen befinden sich solche, deren Formel in Bezug auf die Faktoren der Blumenfärbung der Species $A$. flabellata entsprechen.

Hinsichtlich der Blumenfärbung also, ebenfalls wie hinsichtlich der Form der Sporne, verläuft die Spaltung regelmässig. Wir können schliessen dass hier eine volle Typenreihe in der Spaltung erscheint. 
Die Pflanzen der ersten Hybridengeneration von A. flabellata $\times$ A. chrysantha besitzen hellblaue Blumen (C. C. 486-491) mit hellgelbem Saum der Honigblätter. Es findet hier die Synthese des Anthocyans statt, infolge der Kreuzung von zwei Typen, von denen keiner in seinen Blumen Anthocyan enthält. Ähnliche Erscheinungen wurden, wie bekannt, bei verschiedenen Pflanzen von zahlreichen Autoren beobachtet (Lathyrus (1), Antirrhinum, Matthiola, Mirabilis). Von zwei Faktoren, die bei der Synthese des Anthocyans zusammenwirken, besitzt jede von den beiden gekreuzten Pflanzen nur einen, der deshalb latent bleibt. Wie schon oben erwähnt wurde, enthält $A$. flabellata, ausser dem Faktor $R$ auch den Faktor $F$, der die Farbe des gebildeten Anthocyan bestimmt. Infolgedessen besitzt der entstehende Anthocyan hier immer eine blaue Farbe.

Es muss hervorgehoben werden, dass die Blumenfärbung der $F_{1}$ der beiden hier besprochenen Kreuzungen nicht dieselbe ist, obgleich in beiden Fällen dieselbe A. flabellata zur Kreuzung benutzt wurde. Der Grund dieser Erscheinung liegt in dem Unterschied zwischen der genetischen Konstitution von $A$. chrysantha und $A$. californica. Es spielt hier erstens eine Rolle die Anwesenheit des Anthocyans in den Blumenblätter von $A$. californica im Gegensatz zu seinem Fehlen bei A. chrysantha. In den Pflanzen der $F_{1}$-Generation von $A$. californica A. flabellata ist also der Faktor $R$ homozygot, während er in den $F_{1}$-Pflanzen der zweiten Kreuzung heterozygot erscheint. Dies kann gewissermaassen zu einer Abschwächung den Intensität der Blumenfarbe in der letzten Kreuzung beitragen. Auch in Hinsicht auf die Farbennuance unterscheiden sich untereinander die $F_{1}$-Pflanzen beider Kreuzungen: in der Bastardierung A. flabellata $\times A$. chrysantha hat das Anthocyan eine blaue, nicht eine violette Nuance, obgleich in beiden Bastardierungen die Veränderung seiner Farbe durch denselben von $A$. flabellata eingeführten Faktor bedingt ist. Der Unterschied liegt wahrscheinlich in dem zweiten Faktor, der zur Synthese des Anthocyans beiträgt, nämlich in demjenigen latenten Faktor, der durch die Wirkung von $R$ aktiviert wird und der in $A$. californica mit $C$ bezeichnet wurde. Es ist möglich, dass der entsprechende Faktor von A. chrysantha nicht identisch ist. Zwar führt der betreffende Faktor von A. chrysantha in Zusammenwirkung mit $R$, ebenfalls zur Bildung des Anthocyans, dieser aber ist blass und entwickelt sich in den Blumenknospen dieser Bastarde bedeutend später. Es ist möglich dass die Entwicklung der beiden Substanzen die die Anthocyansynthese bedingen, nicht gleichzeitig stattfindet, nämlich dass die vom Faktor $R$ bewirkte Substanz sich früher zu entwickeln beginnt, während nur 
eine gleich zeitige Entwicklung beider zur Synthese des Anthocyans führen kann. Eine nur kurzdauerende gleichzeitige Wirkung würde zur Bildung einer geringeren Quantität des Pigmentes führen. Die Farbe einer kleineren Menge des gebildeten Pigmentes wurde durch die Wirkung des Faktors $F$ vollständiger modificiert, als in den Blumen von Californica-Bastarden. Da es vermutlich, nach dem Obengesagten in den Pigmentgrundfaktoren der beiden Species Unterschiede zu existieren scheinen, so bezeichne ich den betreffenden Faktor bei $A$. chrysantha mit $C_{1}$.

Zugunsten der oben entwickelten Annahme spricht auch die Existenz einer ganzen Reihe von reinzüchtenden Aquilegia-Gartenvarietäten mit blassgefärbten Blumen, deren Insität ungefähr denjenigen von Chrysantha-Hybriden entspricht. In meiner Kollektion der Aquilegia-Arten und Gartenvarietäten besass ich zwei solche Varietäten, nämlich $A$. azurea hort. mit hellblauen Blumen, und A. azurea fl. roseo mit rosafarbigen Blumen. Bei Bastardierung dieser letzten Varietät mit $A$. flabellata bekommen wir bei $F_{1}$-Hybriden genau dieselbe blassblaue Blumenfärbung, wie diejenige der $F_{1}$-Bastarde von A. flabellata $\times A$. chrysantha, was auch zugunsten der obigen Annahme spricht.

Wie schon oben erwähnt wurde, erscheinen in der Spaltung der Blumenfarben der Bastarde A. flabellata $\times$ A. chrysantha ausschliesslich Pflanzen mit blassgefärbten Blumen. Im Gegensatz zu der Spaltung von der $F_{1}$-Generation von $A$. californica $\times A$. flabellata finden wir hier eine sehr geringe Anzahl Farbentypen (Tabelle VII). Es erscheinen nämlich folgende Blumenfarben :

1) Blaue und hellviolette (bei 23 Pflanzen),

2) Weisse (bei 13 Pflanzen),

3) Hellrosa (bei 2 Pflanzen).

Gelbe Blumen, die dem väterlichen Typus entsprechen, e r s c h e inen überhaupt nicht in der Spaltung, was vermutlich mit der partiellen Sterilität der $F_{1}$-Pflanzen verknüpft ist. Weissblühende Pflanzen dagegen bilden ungefähr $33 \%$ der ganzen (am Leben bleibenden) zweiten Generation, was deutlich beweist, dass die Rekonstruktion der Blumenfarben des mütterlichen Typus keine Hindernisse trifft.

Die erste Gruppe - Pflanzen mit blauen und hellvioletten Blumen, bilden eine nicht einheitliche Gruppe, indem jeder der beiden Faktoren, die bei der Synthese des Anthocyans zusammenwirken, $C_{1}$ und $R$ sowie der Faktor $F$, bald homozygot, bald heterozygot auftreten. 
Einige von dieser Pflanzen lassen in ibren Blumenblättern schwach entwickelte Chromoplasten unterscheiden, die den im heterozygotischen Zustand erscheinenden $Y$ Faktor vermuten lassen.

Pflanzen mit hellrosa (C. C. 0.596) Blumen verdanken ihre Färbung der Anwesenheit der Faktoren $C$ und $R$ in homozygotem oder heterozygotem Zustande. Die Faktoren $F$ und $Y$ sind in ihnen nicht vorhanden.

Die blasse Färbung aller mit Anthocyan gefärbten Blumen in der $F_{2}$ dieser Bastardierung bestätigt die Annahme, dass der Pigmentgrundfaktor $C_{1}$ von $A$. chrysantha mit dem analogen Faktor derjenigen Species, deren Blumen intensiv gefärbt sind, nicht identisch ist.

Die Vererbung der Blumenfarben bei Aquilegia vulgaris ist vor einigen Jahren von Krist offers on (3) studiert worden. Nach diesem Autor werden bei $A$. vulgaris die Blumenfarben von folgenden Faktoren bestimmt:

Der Faktor $B$ ruft hellblaue Färbung („light blue“ C. C. $0 \cdot 466-466$ ) hervor.

Der Faktor $R$ ruft rote, eigentlich rotviolette Färbung („red“ C. C. 571) hervor.

In der Anwesenheit dieser beiden Faktoren, erscheint nach dem Verf. eine dunkelblaue Blumenfärbung (C. C. 428-429), die das Resultat ihres Zusammenwirkens ist. In der Nachkommenschaft der Heterozygoten $\mathrm{RrBb}$ findet eine Spaltung statt, die nach $\mathrm{Kr}$ is t of ferson sich auf das einfache Mendelsche Verhältnis 9:3:3:1 zurückführen lässt, wobei als recessive Farbe weiss erscheint. In dem umfangreichen Material der von Krist offers on in seiner Publikation angegeben ist, entsprechen ungefähr die Zahlen diesem theoretischen Verhältnis, es ist jedoch hervorzuheben, dass in der Spaltung der Typus „red“ stets, nur mit Ausnahme einer unzahlreicher Linie, in grösserer Anzahl, als der Typus „light blue“ erscheint, während theoretisch diese beiden Typen in gleicher Anzahl hätten hervortreten sollen. So z. B. in der Linie, deren Spaltung in Tabelle I (S. 178) vorgestellt ist, finden wir, indem wir die Frequenz pro 16 Individuen berechnen, für „red“ $D+0.60$, für „light blue“ dagegen $D=-020$. In fünf Linien der nächsten Generation (Tabelle III S. 180) beträgt für „red“ $D+0.31$, für „light blue“ dagegen $=-0.66$ bei der Gesamtzahl von 267 Pflanzen (Wahrscheinlich wegen eines Druckfehlers sind die Zahlen +0.24 und -0.36 angegeben.

Aus meinen oben angegebenen Resultaten ist zu schliessen, dass die Anwesenheit von Anthocyanpigmenten in den Blumen von Aquilegia von wenigstens zwei Faktoren bedingt wird. Ein Beweis dafür 
ist die früher erwähnte Tatsache, dass diese Pigmente synthetisch durch die Kreuzung von Pflanzen, die kein Anthocyan haben, dargestellt werden. Ferner enthalten, nach meinen Angaben, rosa und blau gefärbte Blumen dasselbe Anthocyan, dessen Färbung von der Reaktion des Zellsaftes abhängig ist. Da nach Kristofferson die Homozygoten von der genetischen Zusammensetzung $b b R R$ viel intensiver gefärbte Blumen haben, als die „light blue“ Homozygoten $B B r r$, so lässt es uns vermuten, dass bei solchen Pflanzen in den Pigmentgrundfaktoren $C$ und $C_{1}$ Unterschiede vorhanden sind.

Nach meinen Untersuchungen also sollen die genetischen Unterschiede zwischen den beiden Typen "red" und „light blue“ anders formuliert werden, indem ein jeder von den Faktoren Kris t offerson's in der Tat einer ganzen Faktorengruppe entsprechen würde, nämlich :

$$
\begin{aligned}
& B \text { entspricht } C_{1}, R, F \text {; } \\
& R \text { entspricht } C, R, f .
\end{aligned}
$$

Heterozygotische Pflanzen $B b R r$ ("dark blue") würden auf diese Weise meiner Formel $C_{c} C_{1} c_{1} R R F f$ entsprechen. Solch eine Heterozygote würde in ihrer Nachkommenschaft im Verhältnis zu drei Paaren von genetischen Einheiten spalten und für jede 64 Individuen der Nachkommenschaft folgende Typen geben:

36 „dark blue" Pflanzen, von denen 27 die Faktoren $C C_{1} R F$ besitzen;

9 „light blue“ Pflanzen mit den Faktoren $C c_{1} R f$;

15 „red“ Pflanzen mit dunkleren und helleren Blüten; 9 von ihnen besitzen $C C_{1} R f, 3-C c_{1} R f$ und $3-c C_{1} R f$;

4 weissblühende Pflanzen, von denen drei die Faktoren $c c_{1} R F$ und eine $c c_{1} R f$ besitzen.

In dieser Spaltung haben wir, ähnlich wie Krist offers on angibt, 9/16 „dark blue“ Pflanzen und 1/16 weissblühender Pflanzen. Der Unterschied bezieht sich auf die Typen „red“ und "light blue“, die laut meiner Interpretation nicht in gleicher Anzahl erscheinen, sondern einen Überschuss an rotblühenden Pflanzen erweisen, was den Zahlen Krist offerson's besser entspricht. Dies ist aus der Tabelle V ersichtlich.

Zwar könnte man einwenden, dass auch bei dieser Intepretation eine beträchtliche Abweichung von den theoretischen Zahlen zu finden ist, speziell für die Gruppe „red“, deren Pflanzen jetzt in einer zu kleinen Anzahl vorhanden sind. Jedoch ist diese Abweichung geringer, als diejenige, die bei der Erklärung Krist offerson's für die Gruppe „light blue“ zu konstatieren ist; ferner bieten uns ja im 
Tabelle V.

\begin{tabular}{|l||c|c|c|}
\hline \multirow{2}{*}{ Farbentypen } & Obs. & \multicolumn{2}{|c|}{$\begin{array}{c}\text { Theoretisch bei dem } \\
\text { Verhältnis: }\end{array}$} \\
\cline { 2 - 4 } & & $9: 3: 3: 1$ & $36: 15: 9: 4$ \\
\hline Dark blue & 155 & $150 \cdot 19$ & $150 \cdot 19$ \\
Red & 54 & 50.06 & $6: .56$ \\
Light blue & 39 & 50.06 & 37.56 \\
White & 19 & 16.69 & 16.69 \\
\hline
\end{tabular}

Allgemeinen die Spaltungszahlen nur die Möglichkeit, unsere theoretische Erwägungen zu prüfen und zu bestätigen, sie sollen ja nicht selbst eine Quelle für unsere Konceptionen bieten. Meine Interpretation ist nicht ausschliesslich durch Spaltungszahlen begründet, sondern sie findet hauptsächlich ihre Bestätigung in synthetischen Kreuzungen, die zur Entstehung ähnlicher Blumenfarben geführt haben. Ubrigens bemerkt Kristoffers on selbst, dass er keineswegs die von ihm durchgeführte Farbenanalyse als beendigt betrachtet. Bei seinen Untersuchungen von $A$. vulgaris-Varietäten, konnte er nicht diejenigen allgemeinen Formeln aufstellen, die als Resultat des Vergleiches zwischen einer ganzen Reihe von Specieskreuzungen zu schliessen waren.

\section{Koppelungserscheinungen zwischen den Faktoren der Blumenformen und Farben.}

In der früher von mir beschriebenen reciproken Kreuzungen zwischen $A$. vulgaris und $A$. chrysantha (4) wurde eine genotypische Korrelation zwischen der Form der Sporne und der gelben Blumenfarbe der Bastarde konstatiert; sie ist als Folge einer wahrscheinlicher Koppelung zwischen dem Faktor $Y$ und dem Faktor $v$ für die gerade Form der Sporne zu betrachten. Auch in den jetzt beschriebenen Bastardierungen wurde eine Korrelation zwischen den Blumenfarben und Formen gefunden.

Unter den $F_{2}$-Individuen der Kreuzung $A$. californica $\times A$. flabellata erscheinen nicht alle erdenkliche Kombinationen zwischen den Farbentypen und den Blumenformen, die durch die Tabelle II bestimmt sind. Nur der erste Farbentypus, nämlich Pflanzen mit violetten und rotvioletten Blumen sind in allen Formen der Sporne repräsentiert (Tabelle VI). Andere Blumenfarben dagegen sind mit bestimmten Formen der Sporne verbunden. Nämlich die gelbe und orangerote Blumenfarbe erscheint hier in Verbindung mit langen 
Tabelle VI.

\begin{tabular}{|c|c|c|c|c|c|c|c|c|c|c|c|c|c|c|c|c|}
\hline \multirow{2}{*}{ Blütenfarbe von $F_{2}$} & \multicolumn{5}{|c|}{ A } & \multicolumn{5}{|c|}{ B } & \multicolumn{5}{|c|}{$\mathrm{C}$} & \multirow{2}{*}{$\begin{array}{l}\text { Zu- } \\
\text { sammer }\end{array}$} \\
\hline & I & II & III & IV & V & I & II & III $\mid$ & IV & $\mathrm{V}$ & $\mathrm{I}$ & II & III $\mid$ & IV & $\mathrm{V}$ & \\
\hline $\begin{array}{l}\text { Violett und rot- } \\
\text { violett }\end{array}$ & 1 & 12 & 2 & 1 & 2 & 5 & 4 & 12 & 3 & 2 & 1 & 1 & 2 & - & 5 & 53 \\
\hline Orangerot & 1 & 1 & - & - & - & - & 1 & - & - & - & - & - & - & - & - & 3 \\
\hline Helloran & 1 & - & 1 & - & - & - & 1 & - & - & - & - & - & - & - & - & 3 \\
\hline G & - & 1 & - & - & - & - & - & - & - & - & -- & - & - & -- & - & 1 \\
\hline Hellgelb & - & - & 1 & - & - & 1 & - & - & - & - & - & - & - & - & - & 2 \\
\hline $\begin{array}{l}\text { Karminrot } \mathrm{m} . \\
\text { hellgelb }\end{array}$ & - & - & 1 & - & - & 1 & 2 & - & - & - & - & - & - & - & - & 4 \\
\hline Rosa $\mathrm{m}$. hellgelb & 1 & 1 & - & - & - & - & 1 & - & - & - & - & - & 1 & - & - & 4 \\
\hline Ros: & - & - & - & - & - & - & - & 1 & - & - & - & - & - & - & 2 & 3 \\
\hline Weiss & - & - & - & - & - & 1 & - & - & 1 & 1 & - & 1 & 1 & - & 3 & 8 \\
\hline & 4 & 15 & 5 & 1 & 2 & 8 & 9 & $13 \mid$ & 4 & 3 & 1 & 2 & & - & 10 & \\
\hline
\end{tabular}

oder intermediären Spornen deren Form gerade oder gebogen, niemals aber gekrümmt ist (Tabelle VI, Gruppen $A I, I I, I I I$ und $B I, I I$ ). Der Faktor $Y$, welcher mit dem Faktor $v$ gekoppelt zu sein scheint, befinden sich homozygot bei Pflanzen mit orangeroten und hellorangeroten Blumen, in den er teilweise durch das rote Anthocyan verdeckt wird, er erscheint dagegen heterozygotisch in Pflanzen mit karminroten und rosa gefärbten Blumen mit hellgelbem Saum ihrer Honigblätter. Infolge der erwähnten Koppelung besitzen die so gefärbten Blumen lange oder intermediäre und gerade oder schwach gebogene Sporne. Wäre die Koppelung (linkage) zwischen den Faktoren $Y$ und $v$ eine absolute, so würden alle Homozygoten $Y Y$ ausschliesslich gerade Sporne, die denjenigen von A. chrysantha entsprechen oder wenigstens ihnen nahe stehen würden, besitzen (Gruppen $A I, I I)$. Indessen erschienen in der Spaltung zwei Pflanzen von denen die eine die Blumenfarbe von $A$. californica, die andere hellorangerote Blumen besass, mit langen, jedoch leicht gebogenen Spornen (Gruppe $B I I$ ), was vermuten lässt, dass die Koppelung keine vollständige ist. Auch das Erscheinen einer Heterozygote mit rosa gefärbten Blumen und gelbgesäumten Honigblätter in der Gruppe $A I$ beweist dass die erwähnte Koppelung eine partielle ist. Eine Bestätigung dieser Koppelung ist auch das Auftreten von weissen und rosa gefärbten Blumen ohne gelbem Saum, hauptsächlich in den Gruppen mit gekrümmten Spornen, weniger zahlreich dagegen in den Gruppen mit gebogenen Spornen, während sie in den Gruppen 
mit geraden Spornen überhaupt fehlen. Das Material, das mir in der besprochenen Kreuzung zur Verfügung stand, war zu gering, um zu versuchen, die gewonnenen Spaltungszahlen auf theoretische Verhältnisse zurückzuführen. Jedoch ist es zahlreich genug um konstatieren zu können dass in der $F_{2}$-Generation dieser Bastardierung die Rekonstruktion von denjenigen Kombinationen stattfindet, die den beiden elterlichen Typen gleichzeitig hinsichtlich der Blumenformen und Farben entsprechen.

Wenden wir uns nun zur Erörterung der gegenseitigen Abhängigkeit der Blumenfarben und Formen in der zweiten Hybridengeneration der Kreuzung A. flabellata $\times$ A. chrysantha. Wie oben gesagt, konstatieren wir in der Spaltung das Auftreten von nur drei Farbentypen, wobei der väterliche Typus (gelbe Blumenfarbe) fehlt. In der Spaltung der Formen ist der väterliche Typus ebenfalls nicht vorhanden, es erscheinen jedoch in geringer Anzahl ihm nahe stehende Formen (Typus A II, B II, Tabelle IV und VII) mit weissen und blauen Blumen. Die grösste Zahl fällt auf intermediäre Formen, sowie auf Pflanzen, die dem mütterlichen Typus entsprechen oder ihm nahe stehen. (Gruppen IV, V). Bei den drei erwähnten Farbentypen (Tabelle VII) ist kein Zusammenhang mit Formentypen der Sporne wahrzunehmen. Blaue und weisse Blumen treten in allen Gruppen auf. Die rosa gefärbten Blumen sind in einer so geringen Anzahl erschienen, dass es nicht möglich ist, über den Zusammenhang dieser Blumenfarbe mit der Form der Sporne Schlüsse zu ziehen. Der vierte Farbentypus aber, die gelbe Blumenfarbe, die in der Spaltung fehlt, entspricht dem ebenfalls nicht vorhandenen Typus der geraden langen Spornen. Die volle Abwesenheit von diesen Farben- und Formentypus kann nicht durch eine zu geringe Anzahl der $F_{2}$-Individuen erklärt werden,

Tabelle VII.

\begin{tabular}{|c|c|c|c|c|c|c|c|c|c|c|c|c|c|c|c|c|}
\hline \multirow{2}{*}{ Blütenfarbe von $F_{2}$} & \multicolumn{5}{|c|}{$\mathrm{A}$} & \multicolumn{5}{|c|}{ B } & \multicolumn{5}{|c|}{$\mathrm{C}$} & \multirow{2}{*}{$\begin{array}{c}Z u- \\
\text { sammen }\end{array}$} \\
\hline & $\mathrm{I}$ & II & III & IV & $\mathrm{V}$ & I & II & III & IV & $\mathrm{V}$ & 1 & II & III & IV & $\mathrm{V}$ & \\
\hline $\begin{array}{c}\text { Blau } \\
\text { und blauviolett }\end{array}$ & - & 1 & 2 & - & - & - & 2 & 7 & 5 & - & - & - & 1 & 2 & 3 & 23 \\
\hline Rosa & - & - & 1 & 1 & - & - & - & - & - & - & - & - & - & - & - & 2 \\
\hline Weiss & - & 2 & 2 & - & - & - & 1 & - & 1 & - & - & - & - & 4 & 3 & 13 \\
\hline Gelb & - & - & - & - & - & - & - & - & - & - & - & -1 & -1 & -1 & - & - \\
\hline Zusammen & $1-$ & 3 & 5 & 1 & -1 & -1 & 3 & 7 & 6 & $|-|$ & -1 & -1 & 1 & 6 & 6 & 38 \\
\hline
\end{tabular}


indem weissblühende Pflanzen, die sich gegen gelbblühende als recessiv erweisen, auffallend zahlreich repräsentiert sind. Daraus können wir schliessen, dass die Rekonstruktion des väterlichen Typus Störungen trifft, indem wahrscheinlich diejenigen Spo. ren der $F_{1}$-Pflanze, deren Genom dem Chrysantha-Typus entspricht, ausgeschaltet werden. Diese Elimination ist die Ursache einer partiellen Sterilität der $F_{1}$-Pflanzen. Am nächsten dem väterlichen Typus stehen Pflanzen der Gruppe A II, nämlich zwei Pflanzen mit weissen und eine mit blauen Blumen und geraden Spornen, deren Länge von derjenigen von $A$. chrysantha etwas geringer ist. Das Erscheinen der eben erwähnten Typen erlaubt uns zu schliessen, dass es auch hier keine absolute Koppelung zwischen den Faktoren $Y$ und $v$ existiert, da die Kombinationen $y v$ gebildet werden. Jedoch muß man ausdrücklich betonen daß Kombinationen $Y V$, die parallel zu diesen auf dem Wege des Crossing-over gebildet werden sollten, in der Spaltung niemals erschienen sind.

Ein Vergleich der Resultate der Spaltungen beider hier besprochenen Bastardierungen erlaubt uns, die beobachteten Erscheinungen folgenderweise zu gruppieren :

\section{Tabelle VIII.}

\begin{tabular}{|c|c|c|}
\hline $\begin{array}{l}\qquad F_{1} \\
\text { Aussehen der Bastarde: } \\
\text { Fertilität: }\end{array}$ & $\begin{array}{c}\text { Bastarde } A \text {. californica } \\
\times A . \text { flabellata } \\
\text { Intermediäre } \\
\text { Fast normal }\end{array}$ & $\begin{array}{c}\text { Bastarde } A . \text { fIabellata } \\
\times A . \text { chrysantha } \\
\text { Matroklin } \\
\text { Schwach }\end{array}$ \\
\hline $\begin{array}{l}F_{2} \\
\text { Formenreihe in der } \\
\text { Spaltung: }\end{array}$ & vollständig & unvollständig \\
\hline $\begin{array}{l}\text { Auftreten des mütter- } \\
\text { lichen Typus : }\end{array}$ & + & + \\
\hline $\begin{array}{l}\text { Auftreten des väterli- } \\
\text { chen Typus: }\end{array}$ & + & - \\
\hline
\end{tabular}

Auf Grund des obigen Vergleiches zwischen beiden Bastardierungen können wir schliessen, dass es ein deutlicher kausaler Zusammenhang zwischen der normalen Spaltung, in welcher beide elterliche Typen erscheinen, und der normalen Fertilität der $F_{1}$-Pflanzen existiert, während eine unvollständige Formenreihe der Spaltung mit dem Fehlen des väterlichen Typus als Konsequenz der in $F_{1}$ eintretenden Elimination zu betrachten ist. Da in beiden Kreuzungen 
die $F_{1}$-Pflanzen sich im Grad ihrer Ähnlichkeit zu der Mutterpflanze unterscheiden, so enthüllt sich das Problem, ob die partielle Sterilität, die die unvollständige Typenreihe in der Spaltung bedingt auch mit der Matroklinie der Bastarde im Zusammenhang steht. Eine nicht typische Spaltung in den drei erwähnten Bastardierungen mit matroklinen und teilweise sterilen Bastarden, sowie eine typische Spaltung bei fertilen nicht matroklinen Bastarden lässt uns in diesen studierten Fällen einen Zusammenhang zwischen diesen Erscheinungen vermuten. Den hier beobachteten Zusammenhang könnte man folgenderweise kausal begründen:

Bei Bastarden zwischen weniger entfernten Species, bei denen wir keine Matroklinie konstatieren, beeinflusst höchstwahrscheinlich das Eizellplasma der beiden Species in gleicher Weise die Ausbildung der Merkmale der Bastarde. Es existiert hier also infolge der Ähnlichkeit des Plasmas keine Dysharmonie zwischen dem Eizellplasma und dem Genom des väterlichen Typus und infolgedessen erleidet dieser Typus keine Elimination. Dagegen in Bastarden von mehr entfernten Species wird die Fremdheit des Cytoplasmas zur gemeinsamen Ursache beider Phaenomene: der Matroklinie und der Sterilität.

In den vor kurzem publizirten F. v. Wettstein's Untersuchungen an Moosen (6) finden wir interessante Angaben, die mit dem besprochenen Problem eng verknüpft sind. Reciproke Artkreuzungen ergeben nicht identische, sondern matrokline Bastarde (dank einem Unterschied im „Plasmon“). Auch bei Gattungskreuzungen entstehen matrokline Bastarde, die infolge eines stärker ausgeprägten Unterschiedes im "Plasmon" teilweise steril sind. In der Spaltung erscheint der väterliche Typus nicht, was v. Wettstein durch seine Elimination infolge einer Dysharmonie zwischen dem Genom und dem "Plasmon“ zu erklären versucht.

Die hier besprochenen Beobachtungen an Aquilegia-Speciesbastarden bestätigen gewissermassen diese Interpretation. Es steht aber die Frage offen, ob man auf Grund dieser Beobachtungen behaupten kann, dass Aquilegia californica tatsächlich näher $\operatorname{der}$ A. flabellata steht, als $A$. chrysantha. Dafür würde nur ein höherer Fertilitätsgrad ihrer Bastarde sprechen. Dies lässt uns vermuten, dass neben einer weitgegenden Ahnlichkeit der morphologischen Merkmale, Unterschiede einer anderen Art, vielleicht von physiologisch-chemischer Natur vorhanden sein können, die eine Dysharmonie zwischen dem "Plasmon" und Genom bei gewissen Speciesbastarden bedingen können. 


\section{Zusammenfassung.}

In dieser Arbeit wird das Problem des Zusammenhanges zwischen der partiellen Sterilität der Speciesbastarde und dem Fehlen des väterlichen Typus in der $F_{2}$-Generation besprochen. Es werden die Spaltungen von der $F_{2}$-Generation in zwei Bastardierungen, nämlich Aquilegia californica $\times A$. flabellata und A. flabellata $\times A$. chrysantha miteinander verglichen.

Die erste Kreuzung ergibt Bastarde, deren Form und Länge der Sporne eine Kombination der väterlichen und mütterlichen Merkmale vorstellt und deren Fertilität beinahe normal ist, während aus der zweiten Kreuzung Pflanzen entstehen, die in Hinsicht auf die obengenannten Merkmale deutlich matroklin sind und deren Fertilität in hohem Grade abgeschwächt ist. In der $F_{2}$-Generation der fertilen Bastarde von $A$. californica $\times A$. flabellata erscheint in der Spaltung eine vollständige Typenreihe, in der die beiden Elternformen repräsentiert sind. Bei den partiell sterilen Bastarden $A$. flabellata $\times A$. chrysantha dagegen fehlt in $F_{2}$ der väterliche Typus der Spornenform. Die hier eintretende Elimination des väterlichen Typus hat als wahrscheinliche Ursache eine Dysharmonie zwischen dem Eizellplasma und dem Genom, der die Rekonstruktion des väterlichen Typus darstellt.

Der zweite Teil ist einer Analyse der Blumenfarben der Bastarde beider Kreuzungen und dem Zusammenhang zwischen den Blumenfarben und Formen gewidmet.

Das in den Blüten von verschiedenen Aquilegia-Arten erscheinende Anthocyan ist wenigstens von zwei Faktoren bedingt. Zahlreiche Species und Varietäten von Aquilegia, deren Blumen kein Anthocyan enthalten, ergeben infolge von Kreuzungen Bastarde, deren Blumen mit Anthocyan gefärbt sind.

Nämlich bei den Bastarden von A. flabellata $\times A$. chrysantha erscheinen blaue Blumen infolge der Zusammenwirkung des latenten Faktors $C_{1}$ von $A$. chrysantha mit den ebenfalls latenten Faktoren $R$ und $F$, die durch $A$. flabellata eingeführt werden. Die beiden Faktoren $C_{1}$ und $R$ bedingen die Synthese des Anthocyans, der in Anwesenheit des Faktors $F$, der die Veränderung der Anthocyanfärbung verursacht, eine blaue Farbe hat.

In der Spaltung von $F_{2}$ fehlen Pflanzen mit gelben Blumen, was als Folge der oben erwähnten Elimination des väterlichen Typus der Form der Sporne zu betrachten ist, da der Faktor $Y$ für gelbe Blumenfärbung mit dem Faktor $v$ für gerade Sporne gekoppelt ist. 
Die Blumenfärbung von A. californica ist durch die Anwesenheit vom roten Anthocyan und gelber Chromoplasten bedingt, wobei die Blumen orangerote Farbe besitzen. Die Blumenfärbung der $F_{1}$ Bastarde von $A$. californica $\times A$. flabellata ist violett, infolge der Wirkung des von $A$. flabellata eingeführten Faktors $F$, der das rote Anthocyan von $A$. californica ins violette verändert.

Die Blumenfärbung von A. californica ist durch folgende Faktoren bestimmt:

$C$ und $R$ bedingen die Synthese des Anthocyans, der in Abwesenheit des Faktors $F$ rot ist.

$Y$ ist der Faktor für gelbe Blumenfärbung (mit $v$ gekoppelt). Das gemeinsame Hervortreten dieser Faktoren ergibt orangerote Blüten mit gelbem Saum der Honigbläter.

In einer komplizirten, jedoch regelmässig verlaufenden Spaltung der $F_{2}$ Bastarde wurde das Erscheinen beider elterlichen Typen konstatiert, was zugunsten der Annahme spricht, dass in dem ersten der hier besprochenen Fällen die partielle Sterilität als Folge der Elimination bestimmter Typen aufzufassen ist, dass in dem zweiten Fall dagegen die normale Fertilität das Erscheinen einer vollen Typenreihe in der Spaltung bedingt.

Aus dem Botanischen Laboratorium der Freien Universität Polens zu Warschau.

\section{Literaturverzeichnis.}

1) B a te s o n. Mendels Principles of Heredity. Cambridge 1913.

2) Engler u. Prantl. Die natürlichen Pflanzenfamilien. III Teil, 2, Abt. S. 59.

3) Krist of fers on, K. Studies on Mendelian Faktors in Aquilegia vulgaris. Hereditas, 1922, S. 178-190.

4) Skalińska, M. Sur les causes d'une disjonction non typique des hybrides du genre Aquilegia. Acta Soc. Bot. Pol. vol. V, 1928. Note préliminaire dans les Comptes Rend. de la Soc. de Biol. 1927, T. XCVI, p. 1485-6.

5) Skalińska, M. Études sur la sterilité partielle des hybrides du genre Aquilegia. Verh. des V int. Kongr. f. Vererbungsforsch. Zeitschr. f ind. Abst. Supplementband II, 1928.

6) Wettstein, F. v. Über Plasmatische Vererbung sowie Plasma und Genwirkung. Nachr. d. Ges. d. Wiss. Göttingen, 1926. 